\title{
Reexamination of Cariogenesis in Relation to the Distribution of Enamel Lamellae on Crowns of Human Teeth
}

\author{
by \\ Toshihiro ANDO*
}

Abstract

In this study where freshly extracted teeth were used, enamel lamellae were only found on the surfaces other than the occlusal. Contrary to previous studies there was no evidence of lamellae extending to the base of pits or fissures on the occlusal surface from other surfaces. This finding conflicts with a widely known view that lamellae play a role in pit and fissure cariogenesis and suggests, therefore, that the caries susceptibility of pits or fissures has no relationship with lamellae by nature.

\section{Introduction}

Although there have been many studies regarding enamel lamellae, a majority of them are focused on the histological nature of these structures. Since many of these investigators gave diverse interpretations as to the histological nature of lamellae, much controversy and speculation prevail in the literature. As a consequence, a number of different patterns of lamellar morphology and distribution have been reported to occur on crown surfaces of teeth. In spite of the lack of agreement, investigators are of the common opinion that lamellae are most densely distributed cervically and in some cases lamellae occur cervico-occlusally and some of these lamellae extend to the base of pits or fissures where they are connected with one another [1-3]. Also, there are numerous lamellae on the proximal surfaces of crowns, particularly beneath contact points $[1,2,4]$. The reason why the majority of these investigators emphasize the pits and fissures as being most susceptible to caries is due to their physical nature and to their being connected with lamellae which are considered to be important as a caries pathway.

Previously, two methods of investigating the distribution of lamellae have been employed. One method is to prepare cross sections of crowns at various levels and, when the findings gathered from these sections for lamellar distribution are integrated one can then determine the state of their distribution. Most of the previous investigators adopted this method in their studies [3-5]. The other method is to treat specimen teeth with a certain stain and after the crown surface lamellae have been sufficiently stained they are observed in situ [2,6]. Additionally, there is a method in which the enamel is decalcified and the lamellae remaining on the coronal dentin are studied [7].

*安藤利博: Department of Pathology, Nihon University School of Dentistry at Matsudo, 870-1 Sakaecho, Nishi-2, Matsudo, Chiba-ken, 271 Japan (Director: Prof. Dr. Yasuyuki Awazawa). 
A question is raised as to whether all the lamellae observed in extracted teeth are indeed identical to those occurring in vital teeth. The reason for this question is that, in this kind of investigation almost all the specimen teeth have been kept in a fixative for varying lengths of time and even when freshly extracted teeth were used, the usual practice was to fix them for several days before they were examined. Of course, there are cases in which freshly extracted teeth are immediately prepared for specimens by means of a whet-stone without any fixation. Even using this method, however, there is the possibility that lamella-like cracks will be produced in the enamel under mechanical stress associated with grinding. Moreover, it is possible that lamella-like markings (pseudo-lamellae) derived from postmortem cracks may be mistaken for or confused with the putative lamellae existing in vital teeth before extraction. It is known that cracks do occur in enamel after pulp death with the passage of time.

In a previous experiment, in which rabbit incisors were used, certain differences in the development of postmortem enamel cracks have been observed. When these incisors were examined, enamel cracks were found as early as 6 hours after decapitation and these cracks were found to increase with time, with the number of cracks being maximal by 37 hours [8,9]. From these findings, the author was led to believe that, as in the case of rabbit incisors, postmortem enamel cracks would also develop in extracted human teeth. Accordingly, the present study was carried out in which human teeth, immediately after extraction, and preserved in a fixative for a long period of time were compared for distribution of lamellae on the crown. The concern was that the presence or absence of morphological differences between the putative lamellae and cracks (pseudo-lamellae) could be mistaken for lamellae.

\section{Materials and Methods}

Teeth extracted from subjects ranging in age from 30 to 45 years were used. All the teeth used were vital when extracted from patients who showed an advanced degree of periodontal disease. The teeth also showed no apparent exposure of dentin by either attrition or abrasion and exhibited no carious lesions. These specimen teeth were classified into two groups as follows: Group I consisted of fresh teeth within 20 minutes after extraction and Group II contained teeth which had been preserved in $10 \%$ formalin for a period of 6 months to 3 years.

In order to prevent faults and cracks from occurring in the enamel, extraction was done by elevator under infiltration anesthesia without recourse to routine extracting forceps.

For examination purposes, Group I contained a total of 258 teeth consisting of 83 molars, 85 premolars and 90 anteriors; whereas Group II contained 459 teeth comprising 141 molars, 153 premolars and 165 anteriors. Awazawa's method[10] for differentiating the true lamellae from enamel cracks was used. The teeth were first immersed in $0.5 \%$ silver nitrate and left for 2.5 hours. Subsequently, the teeth were transferred to $7 \%$ hydroquinone for 0.5 hour so that the silver nitrate that had permeated into the teeth could be reduced by hydroquinone. Using this procedure a reduced silver film having a black tint was formed on the entire surface 
of the teeth. The treated teeth were then placed in $1 \%$ hydrochloric acid for several minutes to partially decalcify the enamel surfaces. As a result of the partial decalcification the reduced silver film was removed from the enamel surfaces, and the enamel had a white tint and the lamellae were stained black.

The lamellae thus stained were first observed macroscopically and subsequently the very fine lamellae were examined by stereoscopic microscopy. In this manner, efforts were made to determine whether any differences existed in the morphology and distribution of lamellae between Goup I and II.

\section{Results}

Pronounced differences were observed between the lamellae in Gorup I and Group II with regard to their morphology and distributional pattern on crown surfaces. In general the lamellae from the teeth in Group I were found only between the cervical line and occlusal edge in premolars and molars and, in anteriors only between the cervical line and incisal edge. In these cases, although there were numerous lamellae connected to the cervical line, there were no lamellae that reached or extended to the occlusal edge (premolars and molars) or to the incisal edge (anteriors). Moreover, there were no lamellae on the occlusal surfaces of any tooth studied.

Fig. 1 shows enlarged photomicrographs of lamellae as seen on molars and Fig. 2 illustrates lamellae distributed on molars under still greater magnification. Those lamellae seen on the anteriors (Fig. 3), premolars (Fig. 4) and molars (Fig. 5) show similar findings. In other words, lamellae are found only between the cervical line and the occlusal or incisal edge with many of them connecting with the cervical line. It should be noted that no lamellae were found connecting with the occlusal or incisal edge. Lamellae are positioned at a right angle to the cervical line and are usually observed as black lines in large numbers with a majority of short lamellae densely crowded around the neck of the teeth. In addition, there are some lamellae which are free in the region between the cervical line and occlusal or incisal edge. On the whole, lamellae are distributed between the cervical line and occlusal edge in the case of premolars and molars. In anteriors, on the other hand, they are distributed between the cervical line and incisal edge and are particularly densely crowded in the neck of teeth. Even these types of lamellae, which are fairly long, never reach either the occlusal or incisal edge and they terminate invariably at a short distance from it. In molars, premolars and anteriors the lamellae appear to run from the cervical line toward the occlusal or incisal edge. Lamellae which have very short runnings are in the majority and are found to be crowded especially in the neck of premolars and molars. Some of these runnings are found either short or great distances from the cervical line.

Lamellae observed on the teeth preserved in a solution of formalin are presented in $\mathrm{A}$ and $\mathrm{B}$ (anteriors) and $\mathrm{C}-\mathrm{F}$ (premolars) in Fig. 6, and Fig. 7 (molars). They all display the presence of pseudo-lamellae. Many short, fine black lines are observed running at right angles to the cervical line. Long black lines are also seen between the cervical line and the occlusal edge in premolars and molars and between the cervical line and incisal edge in anteriors. These lines do not appear to connect 

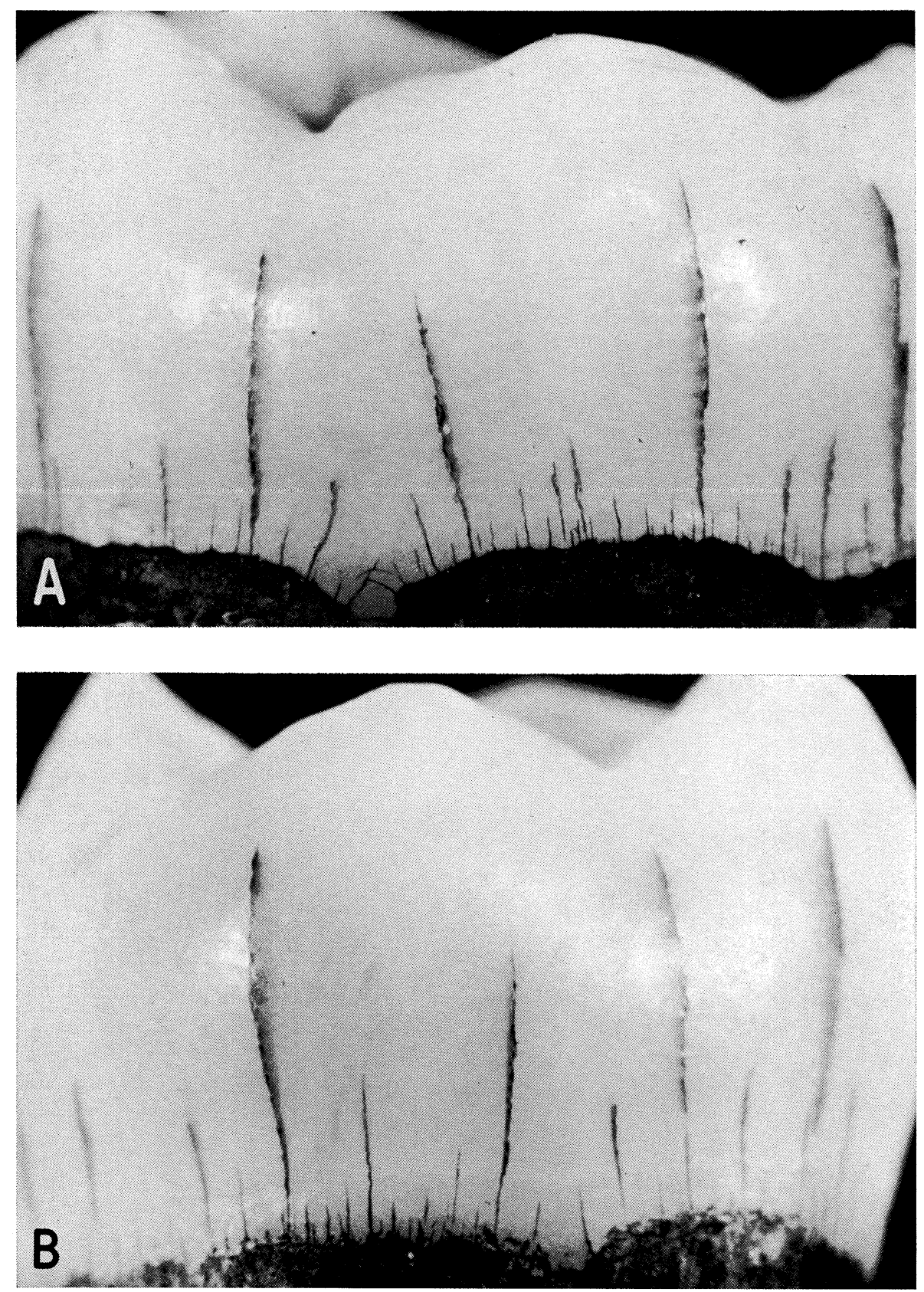

Fig. 1. Enlarged views of lamellae in fresh molars immediately after extraction. Lamellae are densely distributed around the cervical line where short ones are more numerous than long ones. The distribution of lamellae is limited only to a region between the cervical line and the occlusal edge (also refer to Fig. 5). The pictures exhibit lamellae seen on the buccal surface of right mandibular first molar (A) and those on the buccal surface of left maxillary second molar (B). Silver nitrate was used for staining. 

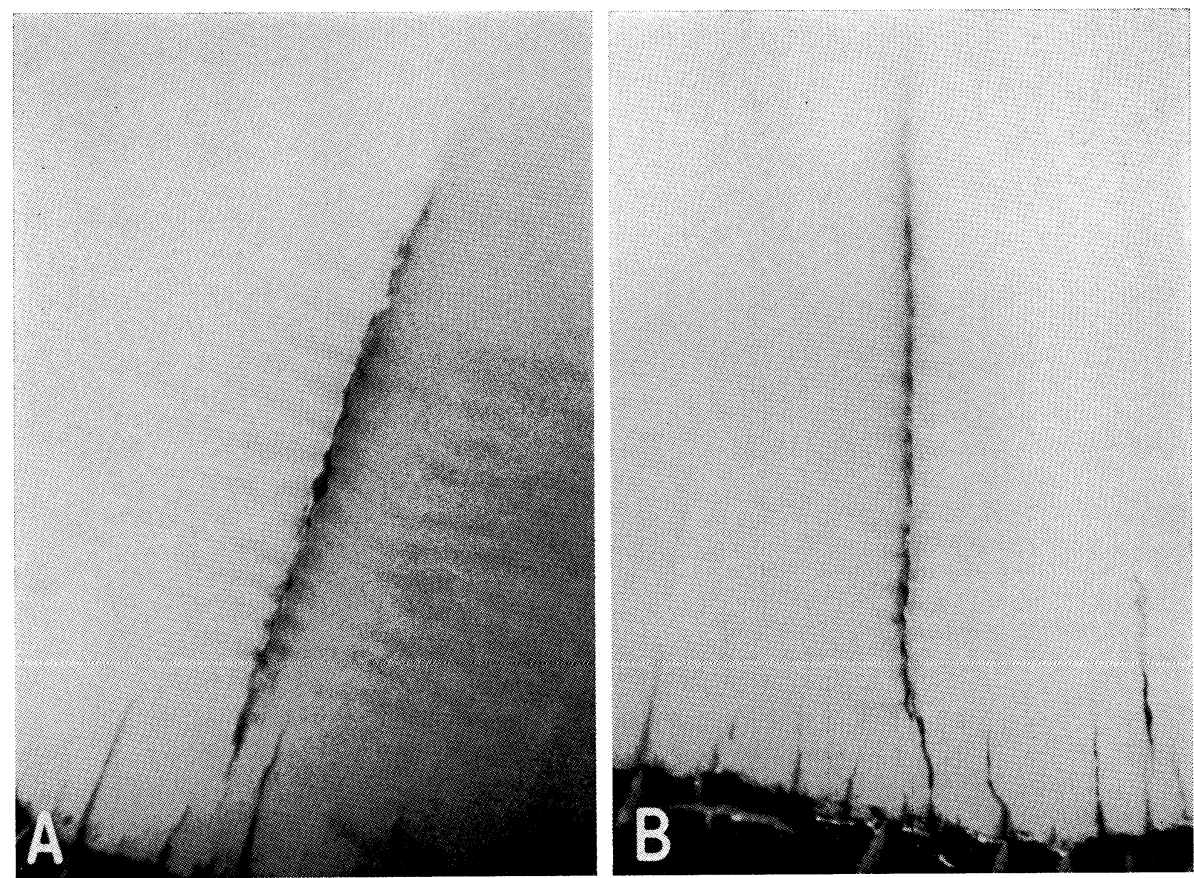

Fig. 2. Highly enlarged views of lamellae observed in the vicinity of the cervical line of fresh molars immediately after extraction. High magnification reveals some lamellae are in a free state in enamel and are not connected with the cervical line. A long lamella (A) and a short one (B) are found in the enamel in a free state respectively. Silver nitrate was used for staining.

with either the occlusal or incisal edge. These findings are similar to those of Group I but in Group II there are additional black lines that occur randomly, running cervico-occlusally and it is difficult to distinguish them from true lamellae. It is assumed that these black lines in the enamel are secondary postmortem changes that are due to alterations in the dental hard tissues after the death of the pulp.

Many lamellae in Group II show black lines that are either thick or slender in their runnings (Fig. 7) and accordingly are distinct from those in Group I. Moreover, some of them are observed to run obliquely in an irregular fashion $(B, D, E$ and $\mathrm{F}$ in Fig. 6, and $\mathrm{A}-\mathrm{C}$ in Fig. 7). In others they are seen as ladder-like forms in which the black lines run parallel to the long axis of the teeth and are crossed almost at right angles ( $\mathrm{H}$ in Fig. 7) by other black lines. Several black lines are connected with each other in a complicated manner having reticular forms ( $D$ in Fig. 6) and further, some are found to possess dendritic branchings ( $E$ and $F$ in Fig. 6). All of these findings are clearly different from those seen earlier in the freshly extracted teeth (Group I). Fig. 8 shows what has been hitherto held to be lamellae observed on the occlusal surfaces of the preserved teeth. A - C in Fig. 8 (premolars) and $\mathrm{D}-\mathrm{F}$ in Fig. 8 (molars) exhibit many black lines running cervico-occlusally. 

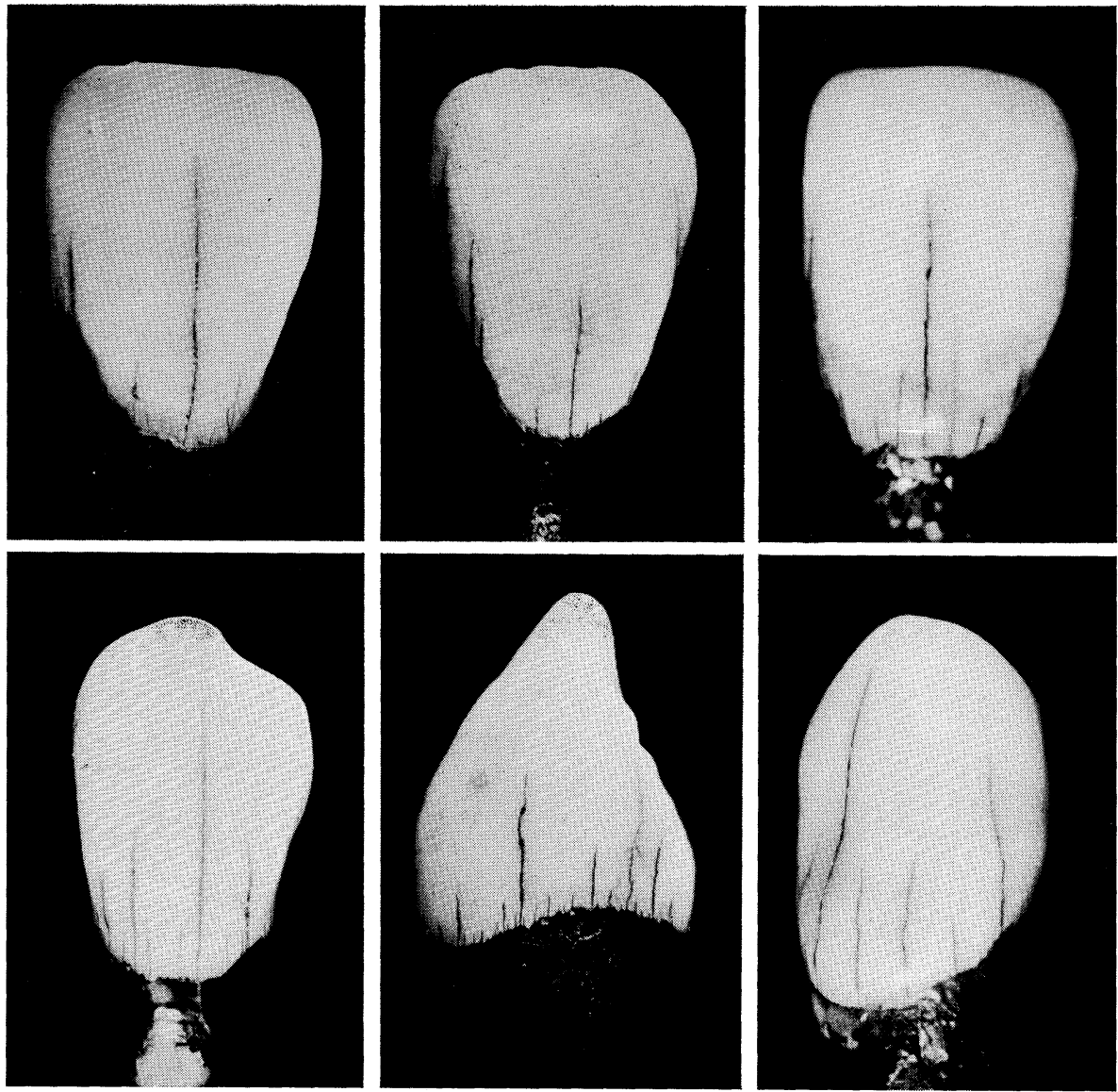

Fig. 3. Lamellae found on fresh anteriors immediately after extraction. Though there are many lamellae reaching the cervical line, none were found to extend to the incisal edge. Silver nitrate was used for staining.

These black lines are observed to be connected with grooves and pits or fissures on the occlusal surface. In addition, there are free lamella-like markings and others that spread radially (E in Fig. 8).

\section{Discussion}

Until recently, there has been no study done which demonstrated that enamel cracks can occur as a postmortem change. This problem was first explained by AwAZAWA and YAMADA [8,9]. These investigators reported that fresh rabbit incisors immediately after decapitation contained no evidence of lamellae. Since pulp death takes place subsequent to decapitation, it follows that the dental hard tissues will eventually lose vitality and will thus result in the death of the teeth themselves.

AwAZAwa and Yamada held the view that cracks would develop in enamel after the death of teeth. Since lamellae are invariably absent in fresh rabbit inci- 

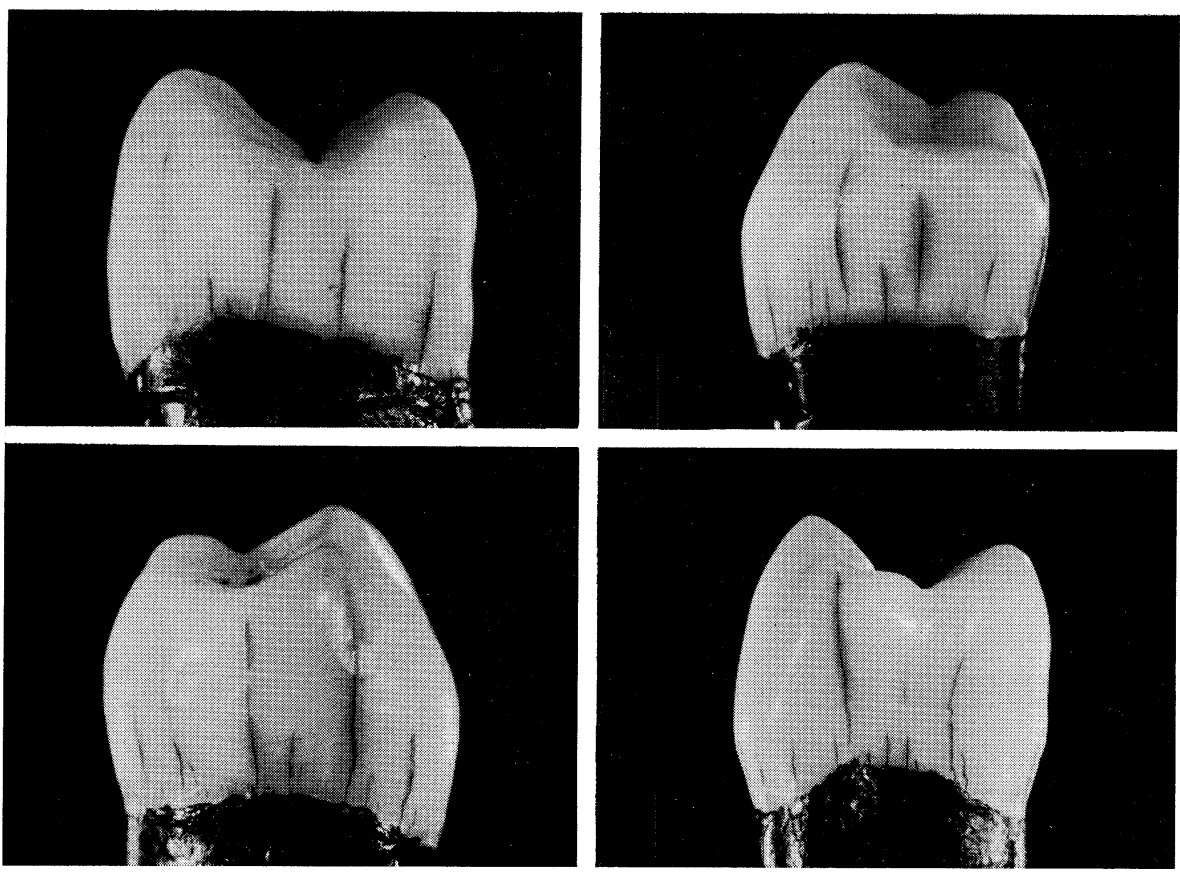

Fig. 4. Lamellae found on fresh premolars immediately after extraction. Lamellae are seen only cervically and never cervico-occlusally. Silver nitrate was used for staining.

sors the use of these incisors was most advantageous in being free of any lamella. For this reason they studied the development of enamel cracks in rabbit incisors as a function of time after death and, as a result, it was established that enamel cracks were found to develop from 6 to 37 hours after the death of teeth.

In this connection, it is interesting that no marked differences were found in the development of these postmortem cracks in teeth under varying conditions such as high or low temperature, immersion in water, burying in the ground or dehydration. For instance, the 197 rabbit incisors placed in water immediately after decapitation were observed for the development of enamel cracks and the development of these cracks, in all of the maxillary and mandibular incisors studied, occurred within 37 hours after death. Using this procedure, enamel cracks were found to develop readily after the death of the teeth. From these findings one can assume that in humans enamel cracks will also develop in extracted teeth proportionately to the passage of time. Although the teeth in Group II showed the presence of lamellae that were connected with pits or fissures, this phenomenon was not observed in the freshly extracted teeth of Group I. Therefore, these cracks in enamel must have developed secondarily as a result of postmortem changes. These lamella-like markings cannot be seen macroscopically and are revealed only after the teeth have been stained.

Previous investigators of lamellae have not indicated the length of time their specimen teeth had been preserved in a fixative after their extraction. It appears 

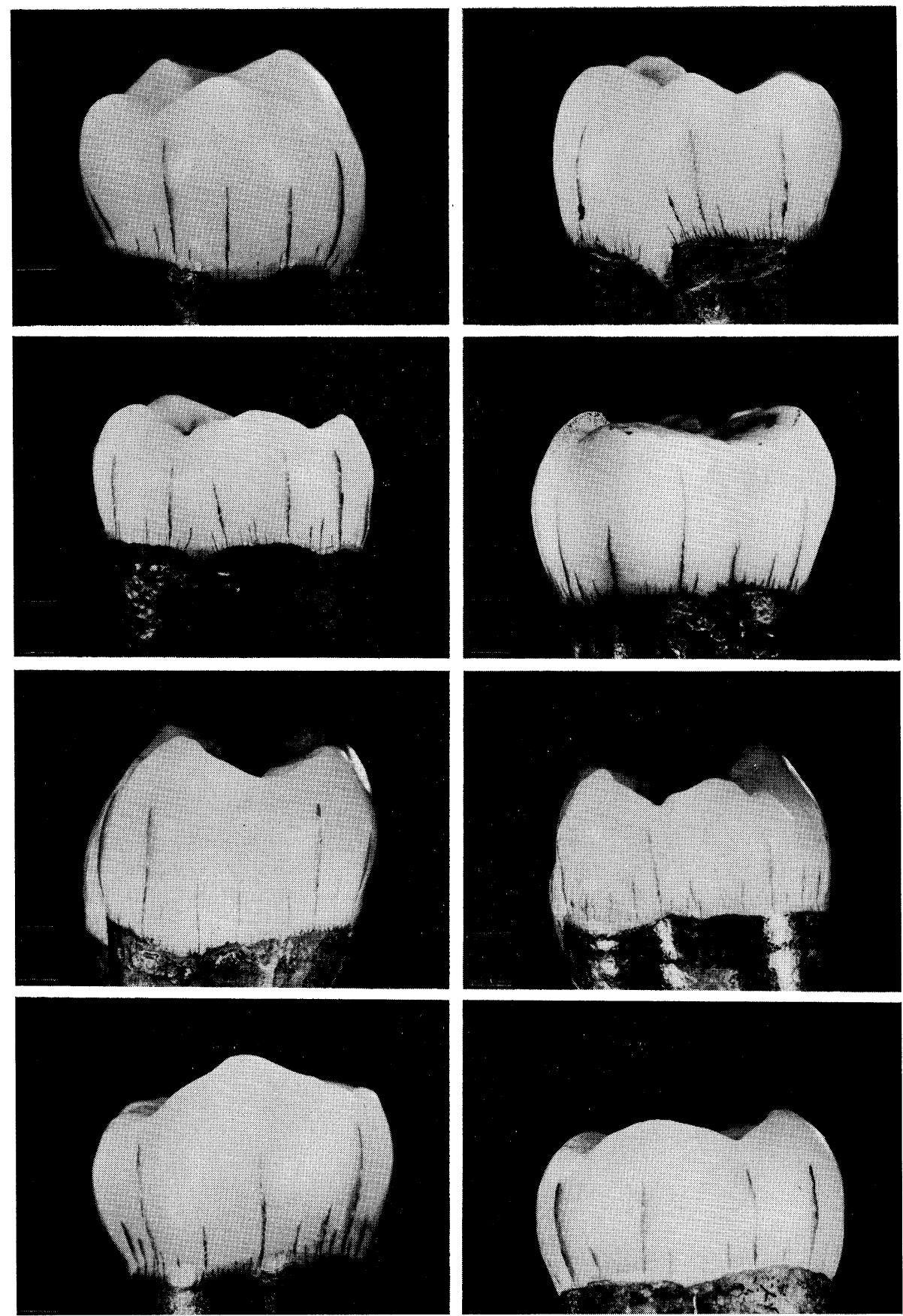

Fig. 5. Lamellae seen in fresh molars immediately after extraction. Lamellae are seen only cervically with many of them connected with the cervical line. Silver nitrate was used for staining. 

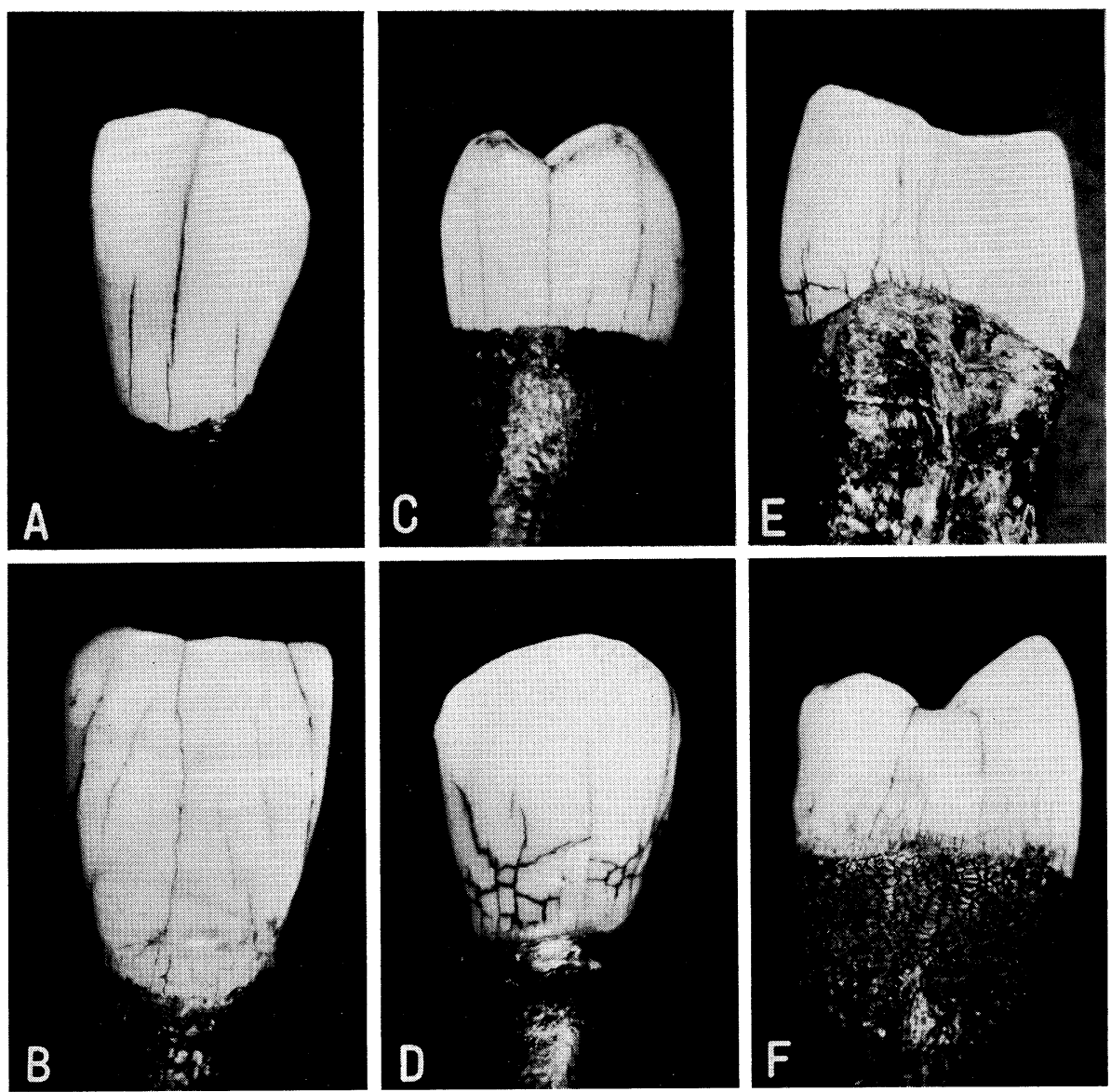

Fig. 6. The effect of preservation of teeth in a fixative from 6 months to 3 years: Lamellae in anterior teeth are seen stretching over both the cervical plane and incisal edge ( $A$ and $B$ ) and some lamellae in premolars extend from the cervical line into the occulusal edge (C). Pseudo-lamellae, displaying a reticular form (D) and a peculiar bifurcation pattern ( $E$ and F), are not seen in the freshly extracted teeth. Silver nitrate was used for staining.

from these studies that this information is necessary in order to evaluate the results reported. It is not rare in some cases that teeth which have been kept in a fixative for 6 months or even a year, have been used for this kind of study. Therefore, in these teeth, the development of enamel cracks could have occurred due to postmortem changes.

Hence postmortem cracks have been regarded as being identical to putative lamellae existing in enamel in previously published reports. This confusion can account for different interpretations regarding the morphology and distribution of lamellae. Lamellae have been reported to be located in the enamel in a state of one thin plate from the cervix to the opposite cervix by way of an occlusal surface [7]. Also other investigations claim lamellae are distributed cervico-occlusally and are 

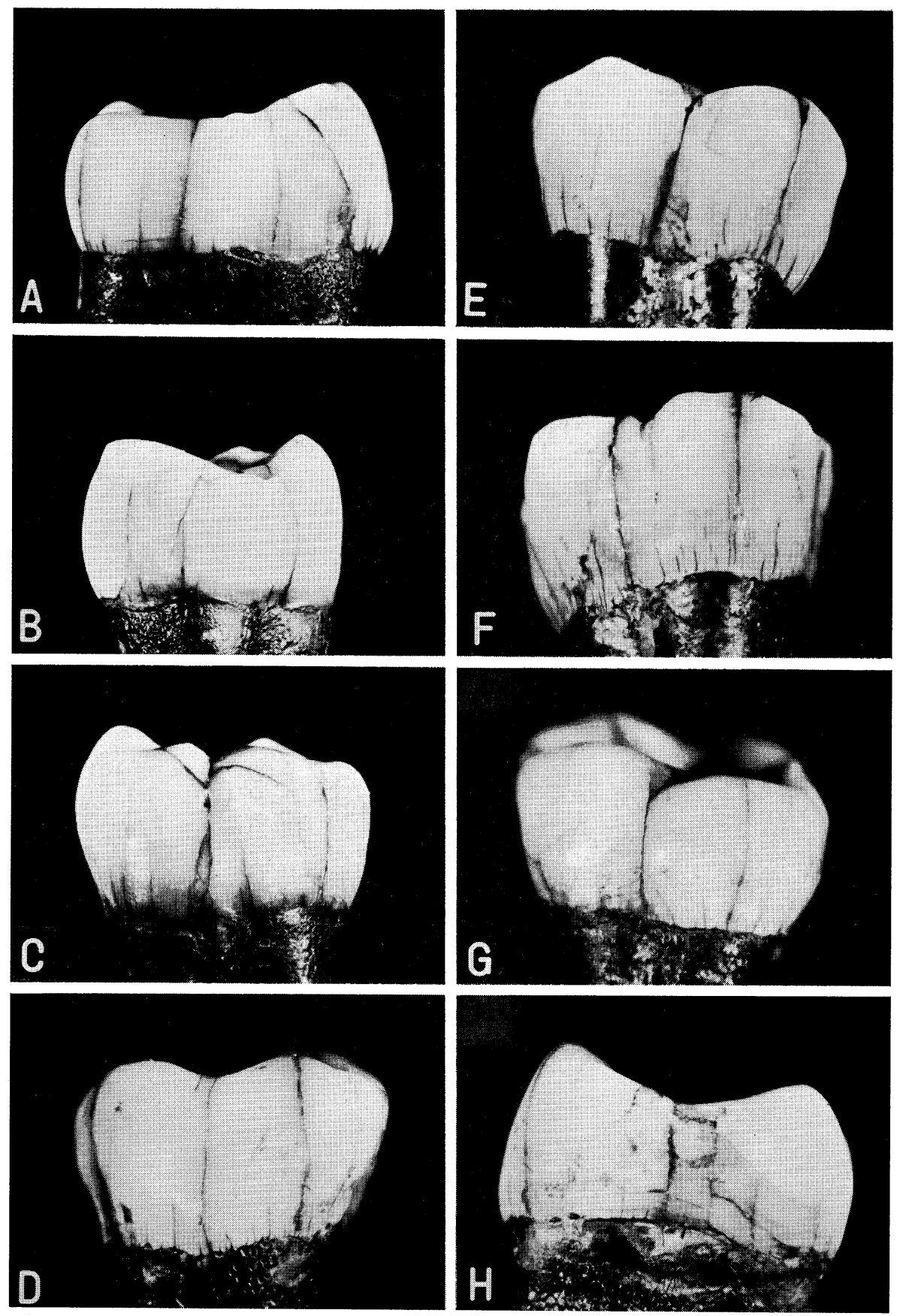

Fig. 7. Pseudo-lamellae in molars preserved in a fixative from 6 months to 3 years. Lamella-like runnings are seen cervico-occlusally, some having peculiar patterns. These types of runnings and distribution are not present in freshly extracted molars. That is, near the neck of the tooth a pseudo-lamella is observed running parallel with the cer- 

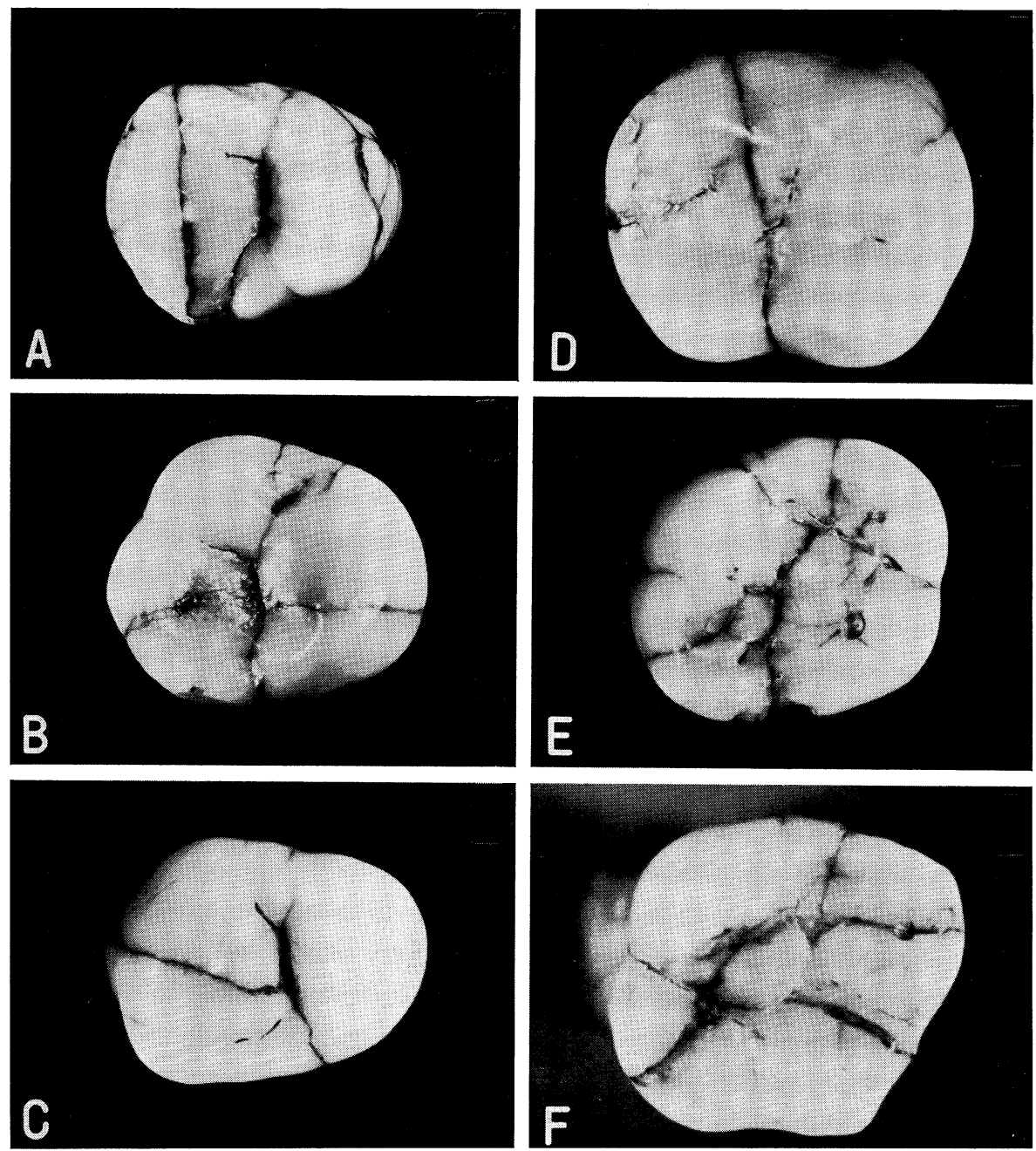

Fig. 8. Pseudo-lamellae on occlusal surfaces of premolars and molars that had been kept in a fixative from 6 months to 3 years. A to $\mathrm{C}$ are concerned with premolars and $\mathrm{D}$ to $\mathrm{F}$ with molars. Some lamella-like runnings are seen in a free state together with those connected with either grooves, pits or fissures and pseudo-lamellae that do not have a definite running direction on the occlusal surface (A to $\mathrm{C}$ ). Sometimes a pseudolamella is seen located in the enamel from the cervix to the opposite cervix by way of the occlusal surface (D). Additionally, a lot of pseudo-lamellae are seen running on the occlusal surface in a very complicated manner (E to F).

vical line (A) and pseudo-lamellae are shown to pass across each other obliquely (B and $\mathrm{C}$ ). Moreover, there are a short pseudo-lamella visible on the occlusal edge (D) and long pseudo-lamellae that are somewhat thick toward the occlusal edge ( $\mathrm{E}$ and F). Some suspected pseudo-lamellae of short running are seen connected with the cervical line not quite at a right angle (G). Some black lines are also seen as ladder-like forms $(\mathrm{H})$. Silver nitrate was used for staining. 
connected with occlusal grooves or with the base of pits or fissures [3,4,11]. Others maintain that although lamellae are generally found on the smooth surface of crowns, in many cases they are connected with pits or fissures [12]. On the other hand, some investigators believe that the majority of lamellae are found connected with fissures [13].

In this study using freshly extracted teeth (Group I), there was no evidence of lamellae on the occlusal surface of crowns. This suggests that caries susceptibility of the pits, fissures or grooves has no relationship with lamellae.

Some postmortem enamel cracks are deeply tinted after staining and their runnings appear as black lines of irregular width and are distinct from the putative lamellae which are of regular widths. However, in some cases this distinction is difficult to make because of their similarities. Other precautions which should be taken in this kind of study, to minimize the formation of postmortem or lamellalike markings, is to avoid the use of extraction forceps and to take great care in preparing ground sections of teeth. It is recommended that the method proposed by AWAZAWA [10] should be followed in the preparation of ground sections and lamellae should be stained within a few hours after extraction in order to obtain meaningful results.

\section{Conclusions}

Two groups of specimen teeth, one consisting of teeth immediately after extraction (Group I) and the other, teeth kept in a fixative anywhere from 6 months to 3 years after extraction (Group II), were examined for the presence of enamel lamellae. After staining with silver nitrate the lamellae in Group I were found only on surfaces other than the occlusal. In Group II, on the other hand, the lamellae were not only seen as in Group I but additionally many lamellae occurred running on the occlusal as well as the other surfaces. Therefore, those lamellae seen in Group II running occlusaliy or cervico-occlusally are considered to be lamella-like markings (pseudo-lamellae) which have developed as postmortem cracks following extraction and storage in a fixative. This distribution of lamellae and pseudo-lamellae has no bearing on the cariogenesis on the occlusal surface.

\section{Acknowledgments}

The author is deeply grateful to Professor Yasuyuki Awazawa, Department of Pathology and Oral Pathology at Nihon University School of Dentistry at Matsudo, for his constant guidance and encouragement. Thanks are also extended to the staff of the Department who helped the author, directly or indirectly, in the execution of the present study.

\section{References}

[1] Gottlueb, B.: Dental Caries. Its Etiology, Pathology, Clinical Aspects and Prophylaxis, 1st ed. Philadelphia: Lea \& Febiger, 1947, pp. 39-40.

[2] Hodson, J. J.: An investigation into the microscopic structure of the common forms of enamel lamellae with special reference to their origin and contents. I. General considerations and Development, Oral Surg. Oral Med. Oral Pathol., 6, 305-317, 1953.

[3] Hodson, J. J.: An investigation into the microscopic structure of the common forms of enamel 
lamellae with special reference to their origin and contents. II. The form and contents of lamellae in unerupted but calcified crowns with special reference to hypoplastic lesions, Oral Surg. Oral Med. Oral Pathol., 6, 383-398, 1953.

[4] Gottlieb, B.: Untersuchungen über die organische Substanz im Schmelz menschlicher Zähne. Ein Beitrag zur Aetiologie der Zahnkaries, Oesterreich.-Ungar. Vielterjar. Zahnhkd., 31, 19-33, 1915.

[5] Hodson, J. J.: An investigation into the microscopic structure of the common forms of enamel lamellae with special reference to their origin and contents. III. Lamellae in erupted teeth with special reference to the form and contents of classical type, Oral Surg. Oral Med. Oral Pathol., 6, 495-515, 1953.

[6] Sognnaes, R. F.: The organic elements of the enamel. IV. The gross morphology and the histological relationship of the lamellae to the organic framework of the enamel, J. Dent. Res., 29, 260-269, 1950.

[7] Meyer, W.: Normale Histologie und Entwicklungsgeschichte der Zähne des Menschen. A. Normale Histologie, I. Schmelz, Zweite Auflage. München: Carl Hanser Verlag, 1951, S. 26.

[8] Awazawa, Y. and Yamada, H.: Experimental observations of the occurrence of the postmortem cracks in tooth enamel (A), J. Hihon Univ. Sch. Dent., 2, 198-210, 1960.

[9] Awazawa, Y. and Yamada, H.: Experimental observations of the occurrence of the postmortem cracks in tooth enamel (B), J. Nihon Univ. Sch. Dent., 3, 23-37, 1960.

[10] Awazawa, Y.: A method for differentiating true lamellae from enamel cracks, Acta Anat. Nippon., 27, 141-147, 1952. (Japanese text with English abstract)

[11] Manley, E. B. and Hardwick, J. L.: Caries of the enamel, Brit. Dent. J., 91, 36-43, 1951.

[12] Faber, F.: Die Lamellen des Zahnschmelzes, Deutsch. Monatsschr. Zahnhkd., 43, 354-357, 1925.

[13] Brammer, F. und Brauer, K.: Über organische Substanz im Schmelz menschlicher Zähne, Deutsch. Monatsschr. Zahnhkd., 44, 473-494, 1926. 